

\title{
Croissance du frene, structure de l'appareil photosynthétique et disponibilité en eau
}

Noël Le Goff, André Granier, Jean-Marc Ottorini, Karine Le Gall, Marianne Peiffer

\section{- To cite this version:}

Noël Le Goff, André Granier, Jean-Marc Ottorini, Karine Le Gall, Marianne Peiffer. Croissance du frene, structure de l'appareil photosynthétique et disponibilité en eau. Revue forestière française, 1995, 67 (S), pp.156-164. 10.4267/2042/26708 . hal-03444297

\section{HAL Id: hal-03444297 \\ https://hal.science/hal-03444297}

Submitted on 23 Nov 2021

HAL is a multi-disciplinary open access archive for the deposit and dissemination of scientific research documents, whether they are published or not. The documents may come from teaching and research institutions in France or abroad, or from public or private research centers.
L'archive ouverte pluridisciplinaire HAL, est destinée au dépôt et à la diffusion de documents scientifiques de niveau recherche, publiés ou non, émanant des établissements d'enseignement et de recherche français ou étrangers, des laboratoires publics ou privés. 


\title{
CROISSANCE DU FRÊNE, STRUCTURE DE L'APPAREIL PHOTOSYNTHÉTIQUE ET DISPONIBILITÉ EN EAU
}

\author{
N. LE GOFF - A. GRANIER - J.-M. OTTORINI \\ Karine LE GALL - Marianne PEIFFER
}

Cet article s'appuie en partie sur les résultats obtenus par Karine Le Gall et Marianne Peiffer lors de leur stage de maitrise et post-maitrise portant sur létude du fonctionnement écophysiologique du Frêne en peuplement dans le cadre du dispositif experimental installé en forêt d'Amance (Meurtheet-Moselle).

Cette étude s'insère dans un programme de recherches visant à élaborer un système de simulation informatisé permettant de rendre compte de la croissance et du développement de peuplements équiennes de Frêne (Fraxinus excelsior L.) soumis à des traitements sylvicoles variés (Le Goff et Ottorini, 1990). Un tel système devrait permettre de combler en partie le manque de données expérimentales sur les effets des traitements sylvicoles pour cette espèce (densités de plantation, éclaircies, ...) et ainsi de guider sa sylviculture. Ce futur système de simulation reposera sur un ensemble de relations de croissance et de développement de l'arbre (modèle) mises en œuvre dans le cadre d'un programme informatique qui gérera simultanement la croissance et le développement de l'ensemble des arbres d'une placette forestière.

Le modèle de croissance individuel envisagé repose sur le développement des houppiers des arbres (figure 1, p. 157). Dans ce modèle, les dimensions du houppier sont établies à partir d'une relation liant l'élongation des branches principales à celle de la tige et de relations caractérisant la forme et le port des branches. De telles relations ont pu être établies à partir d'analyses de tiges et de branches sur un échantillon d'arbres abattus, ce qui a permis d'en déduire une équation générale décrivant le profil du houppier du Frêne (Cluzeau et al., 1994) (1). En réalité, l'élongation des branches ne peut avoir lieu que si aucun obstacle (houppier d'un arbre voisin) ne s'y oppose ; la limitation au développement du houppier due à la concurrence sera prise en compte par le système de simulation envisagé grâce à la représentation spatiale des houppiers dans la mémoire de l'ordinateur. Une fois établies les dimensions du houppier, le volume occupé par le feuillage une année donnèe, ou "volume foliaire", est obtenu comme le produit de la surface de projection au sol du houp- 
pier et de l'élongation de la tige, suivant le procédé employé pour le Douglas (Ottorini, 1991). L'accroissement volumique de la tige apparaît alors comme une fonction du volume foliaire et de la surface latérale de la tige (ou surface cambiale) : une telle relation a pu être établie pour le Frêne par l'analyse rétrospective de la croissance volumique annuelle des arbres-échantillon abattus et la reconstitution des dimensions passées de leur houppier (Ottorini et al., 1996). Par ailleurs, on a pu vérifier que les variations de la surface du cerne annuel suivaient sensiblement la loi de Pressler, ce qui permet de répartir l'accroissement volumique annuel le long de la tige suivant ce schéma (Ottorini et al., 1996).

Dans un tel modèle de croissance, c'est l'élongation de la tige qui contrôle l'ensemble des processus de croissance et de développement de l'arbre (cf. figure 1, ci-dessous). La possibilité de mieux contrôler ces processus a ainsi été envisagée par la prise en compte des interactions entre ces processus, les facteurs de l'environnement et la concurrence entre arbres, notamment au travers des variations morphologiques et de fonctionnement de l'appareil photosynthétique de l'arbre (figure 1). Pour cela, il a été décidé de mettre en place une expérimentation portant sur des frênes diversement concurrencés et soumis à des variations des facteurs environnementaux (climat annuel notamment) susceptibles de modifier la disponibilité en eau pour les arbres, facteur particulièrement déterminant de la croissance du Frêne (Aussenac et Lévy, 1992 ; Lévy et al., 1992).

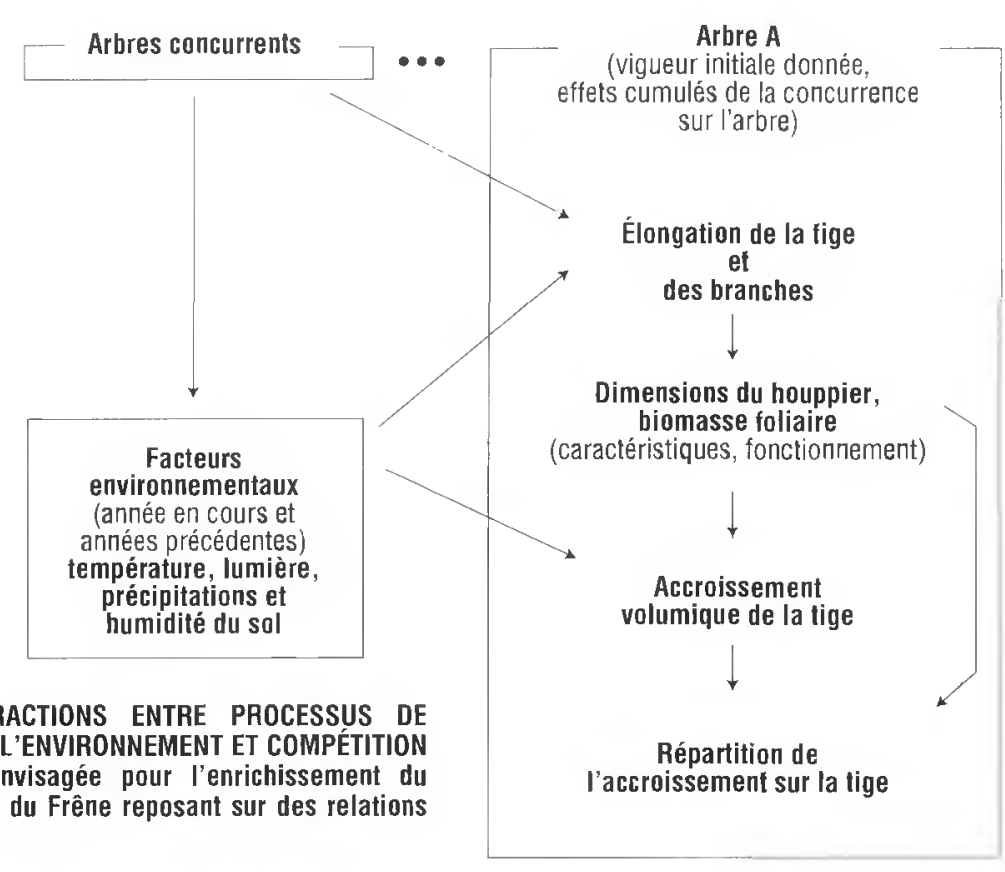

Figure 1

MODÉLISATION DES INTERACTIONS ENTRE PHOCESSUS DE CROISSANCE, FACTEURS DE L'ENVIRONNEMENT ET COMPÉTITION ENTRE ARBRES, felle qu'envisagée pour l'enrichissement du modèle de croissance actuel du Frêne reposant sur des relations de type dendrométrique
Arbre A

(vigueur initiale donnée sur l'arbre)

longation de la fige

des branches

imensions du houppier. aractéristiques, fonctionnement

Accroissement valumique de la tige

Répartition de l'accroissement sur la tige

\section{MATÉRIEL ET MÉTHODES}

Les frênes étudiés appartiennent à un peuplement équienne installé naturellement, après coupe à blanc du taillis-sous-futaie pré-existant, dans une parcelle de la forêt domaniale d'Amance (Meurtheet-Moselle) située près du Centre INRA de Nancy. Ce peuplement, âgé d'une vingtaine d'années, installé sur sol brun lessivé, comporte à la fois des rejets de souche et des arbres de franc pied appartenant à diverses espèces feuillues. Une placette de 10 ares environ, riche en frênes issus de 
semis, y a été délimitée et un échantillon d'une vingtaine de frênes couvrant une grande amplitude de statuts concurrentiels sélectionné (Le Goff et Ottorini, 1996).

La croissance et le fonctionnement écophysiologique des arbres échantillonnés seront étudiés sur plusieurs années successives de manière à observer, si possible, les effets de différentes conditions climatiques. En 1993, première année d'expérimentation, seuls la croissance et le développement des frênes ont été observés. En 1994, aux mesures de croissance ont été associées des mesures concernant le fonctionnement hydrique et photosynthétique pour un sous-échantillon d'arbres.

Chaque année, les arbres étudiés sont l'objet des mesures dendrométriques suivantes (cf. Le Goff et Ottorini, 1996) :

- accroissement radial hebdomadaire de la tige à $1,30 \mathrm{~m}$, du début à la fin de la saison de végétation, grâce à des rubans micro-dendrométriques entourant la tige,

— circonférence de la tige à différents niveaux $(0,30 \mathrm{~m} ; 1,30 \mathrm{~m}$; base du houppier ; mi-distance entre 1,30 $\mathrm{m}$ et la base du houppier), avant et à la fin de chaque saison de végétation, ces mesures permettant d'évaluer l'accroissement volumique annuel de la tige,

- surface de projection du houppier et hauteur totale de la tige au début de l'expérience, puis a la fin de chaque saison de végétation, permettant d'en déduire le "volume foliaire" annuel,

- biomasse foliaire totale (poids sec des feuilles sans leur pétiole), par recueil des feuilles grâce à des filets installés sur chaque arbre (cf. photo ci-dessous),

- surface foliaire et poids sec d'un échantillon de feuilles, permettant d'en déduire la surface spécifique moyenne des feuilles des arbres étudiés et ainsi d'estimer la surface foliaire totale des arbres.

Pour ce qui concerne le fonctionnement hydrique, un sous-échantillon de 9 arbres, de divers statuts concurrentiels, fait l'objet de mesures de transpiration en continu tout au long de la saison de végé-

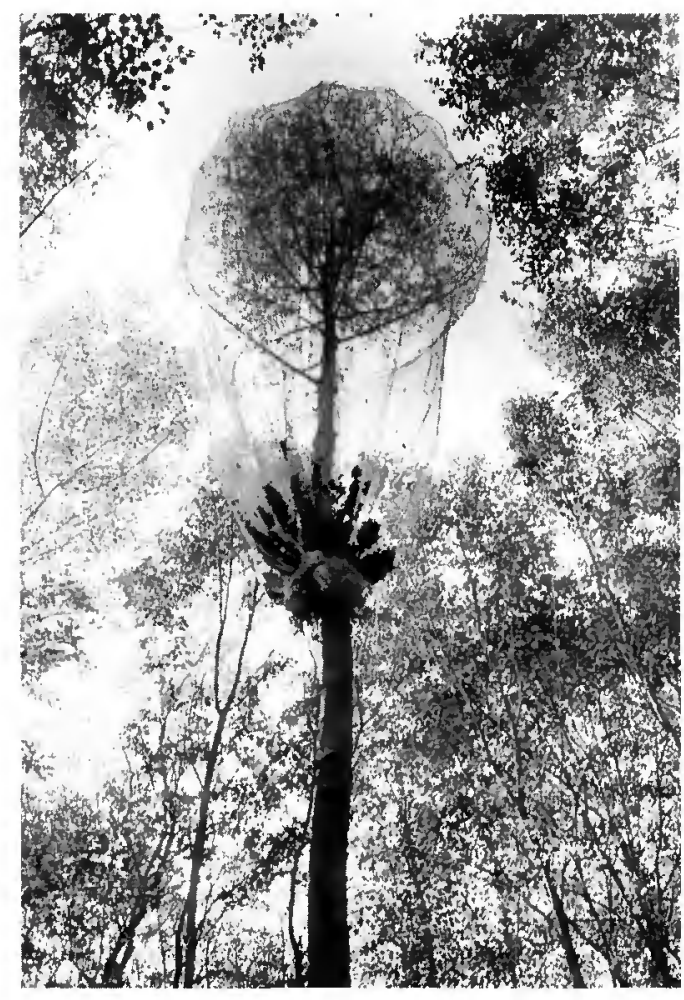

tation, grâce à des capteurs installés sur le tronc mesurant le flux de sève circulant dans l'arbre. Par ailleurs, le potentiel hydrique foliaire de base de ces arbres est mesuré régulièrement tout au long de la saison de végétation (au pas de temps de 15 jours environ), de façon à suivre l'état hydrique des arbres au fur et à mesure du dessèchement du sol.

Mafériel expérimental : vue partielle du peuplement expérimental en forêt domaniale d'Amance (Meurfhe-ef-Moselle) monfrant certains des frênes éfudiés avec le filef posé à l'automne pour recueillir le feuillage ef estimer ainsi la biomasse et la surface foliaires de chaque arbre Photo R. CANTA 
Parallèlement à ces mesures, différentes techniques de caractérisation de I'humidité et du potentiel hydrique du sol sont utilisées pour suivre l'évolution de l'état hydrique du sol (Le Gall, 1994).

Sur un plus petit nombre d'arbres au houppier accessible grâce à des échafaudages, des mesures plus détaillées sont réalisées de manière à pouvoir analyser la structure de l'appareil photosynthétique (croissance et développement des branches, répartition du feuillage) et le fonctionnement du feuillage (transpiration, photosynthèse) en relation avec les paramètres microclimatiques. Les données ainsi obtenues, non relatées ici, ont tait l'objet pour certaines d'une première exploitation, notamment au plan écophysiologique (ct. Le Gall, 1994 ; Peitfer, 1995).

\section{RÉSULTATS ET DISCUSSION}

\section{Biomasse et surface foliaires}

Les données rapportées sont celles de l'année 1993 ; elles concernent 14 arbres sur les 17 étudiés la première année de l'expérimentation, trois arbres n'ayant pu ètre équipés de filets comme les autres. La biomasse foliaire mesurée, qui varie entre 0,3 et $2,9 \mathrm{~kg}$, est proportionnelle au volume foliaire estimé (Le Goff et Ottorini, 1996) (figure 2, ci-dessous). Ce volume foliaire, qui est le volume compris entre les enveloppes du houppier de début et de fin de saison de végétation, semble ainsi bien rendre compte des variations de biomasse foliaire entre arbres, liées d'une part aux dimensions initiales du houppier (fonction des conditions passées de croissance et de concurrence de chaque arbre), mais aussi au potentiel de croissance annuel de chaque arbre traduit dans le volume foliaire par l'accroissement en hauteur.

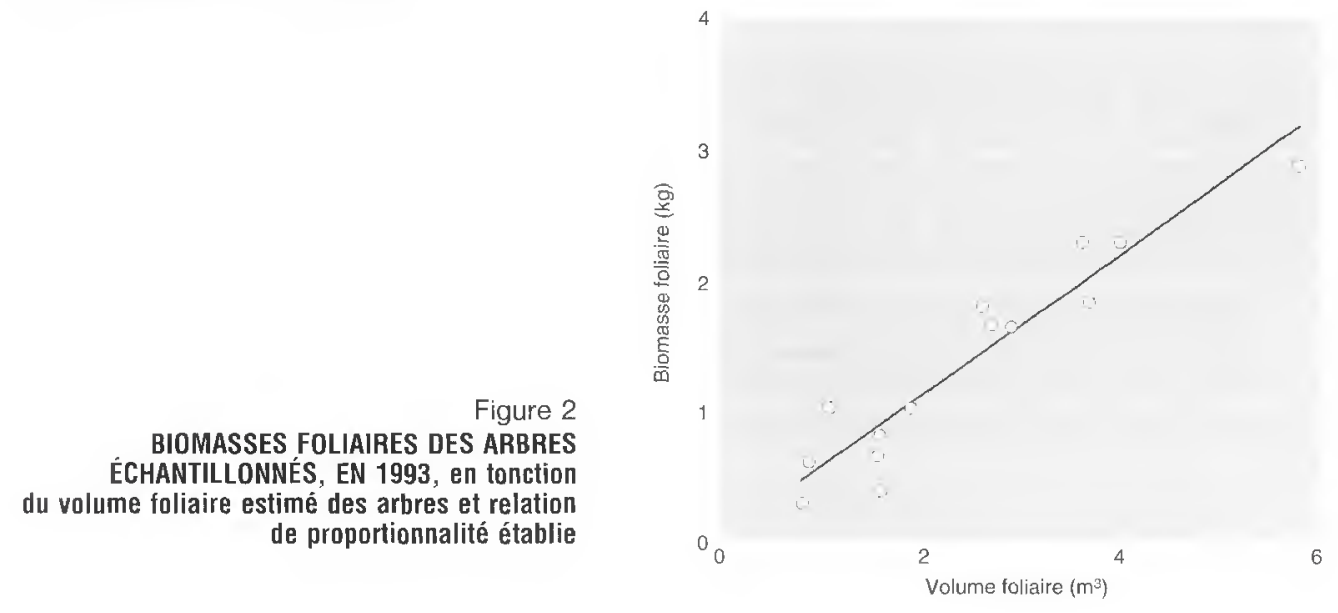

La surface foliaire de chaque arbre a été obtenue comme le produit de sa biomasse foliaire par la surface spécifique moyenne de l'échantillon de feuilles mesuré, égale à $158,85 \mathrm{~cm}^{2} / \mathrm{g}$. La surface foliaire calculée est donc proportionnelle à la biomasse foliaire; elle varie de $4,8 \mathrm{~m}^{2}$ à $45,70 \mathrm{~m}^{2}$ et dépend linéairement, comme la biomasse foliaire, des dimensions du houppier (Le Goff et Ottorini, 1996). Des variations de la surface spécifique foliaire ont cependant pu être notées, variations entre arbres mais aussi pour un même arbre suivant le niveau du feuillage dans le houppier ; un échantillonnage plus important prenant en compte ces sources de variation a ainsi été prévu pour les années ultérieures, de façon à améliorer l'estimation de la surface foliaire totale.

L'indice foliaire (LAI) a été calculé pour chaque arbre comme le rapport de sa surface foliaire totale à la surface de projection au sol de son houppier. II varie de 2 à $6 \mathrm{~m}^{2}$ de teuillage environ par $\mathrm{m}^{2}$ 


\section{N. LE GOFF - A. GRANIER - J.-M. OTTORINI - Karine LE GALL - Marianne PEIFFER}

de surface de projection au sol pour les arbres étudiés. Les variations de LAI ont pu être mises en relation avec le statut concurrentiel des arbres, évalué par la hauteur relative du houppier (figure 3 , ci-dessous). Ainsi, moins l'arbre est concurrencé (la hauteur relative du houppier augmente), plus l'indice foliaire est élevé (en moyenne) ; il semble cependant que l'indice foliaire atteigne un maximum pour des arbres moyennement concurrencés (houppier représentant environ $45 \%$ de la hauteur


totale de l'arbre) et qu'il diminue lorsque les arbres deviennent plus faiblement concurrencés. Ces résultats pourront être confirmés grâce aux observations des années ultérieures, en particulier pour les arbres dont le houppier aura pu se développer librement grâce aux dégagements effectués.

Figure 3

VARIATIONS DE L'INDICE FOLIAIRE (LAI) en fonction du statut concurrentiel des arbres mesuré par la hauteur relative du houppier (rapport de la longueur du houppier à la hauteur totale de l'arbre) en 1993

\section{Croissance volumique des arbres}

À partir des observations faites en 1993, on a pu établir que l'accroissement volumique des arbres augmentait quasiment proportionnellement avec la biomasse foliaire (Le Goff et Ottorini, 1996). L'accroissement volumique de la tige dépend aussi de l'indice foliaire des arbres, comme le montre la figure 4 (ci-dessous) : l'efficience du feuillage, ou volume de bois de tige produit par unité de biomasse foliaire, décroît avec l'indice foliaire de l'arbre. Ainsi, si l'on considère la relation mise en évidence entre indice foliaire et statut concurrentiel des frênes (cf. figure 3, ci-dessus), il apparaît que plus les arbres sont concurrencés, meilleure est leur efficience foliaire; les arbres peu concurrencés (houppier représentant près de la moitié de la hauteur totale de l'arbre et au-delà) retrouveraient cependant une meilleure efficience foliaire. Ce résultat est à rapprocher de ceux obtenus pour d'autres espèces, montrant que l'accroissement volumique des arbres augmente, pour des dimensions


de houppier données, avec l'état de concurrence de l'arbre (Ottorini, 1991 ; Le Goff et Ottorini, 1995).

Figure 4

RELATION ENTRE L'ACCROISSEMENT VOLUMIQUE ANNUEL DE LA TIGE PAR UNITÉ DE BIOMASSE FOLIAIRE (EFFICIENCE FOLIAIRE) ET L'INDICE FOLIAIRE DES ARBRES EN 1993 


\section{Fonctionnement hydrique}

Tout d'abord, on a pu constater que la transpiration des arbres (mesurèe par le liux de sève cumulé) augmentait fortement avec la surface foliaire, donc avec les dimensions du houppier (figure 5, cidessous) : les arbres au houppier le plus développé sont ceux qui transpirent le plus et qui ont aussi la plus forte croissance. Ceci permet d'expliquer que l'intensité de la transpiration diminue avec l'ètat de concurrence de l'arbre, c'est-à-dire lorsque la hauteur relative du houppier diminue ; les figures $6 a$ et $6 b$ (ci-dessous) montrent ainsi les variations de la densité de flux de sève avec le statut concurrentiel des arbres pour la transpiration journalière d'une part, pour la transpiration saisonnière d'autre part.

Figure 5

TRANSPIRATION DES FRÊNES ET SURFACE FOLIAIRE : relation entre le flux de sève cumulé (du 19 juin au 13 aaûl 1994) et la surlace foliaire de 9 Irênes en conditions naturelles

Figure 6

\section{TRANSPIRATION DES FRÊNES ET STATUT}

\section{CONCURRENTIEL}

6A Variations journalières de la densité de flux de sève pour 9 frênes de slałufs concurrentiels variés. Amance : 1994, jour 202 (21 juillet)

\section{Statut concurrentiel des frênes} 2,0

(mesuré par la hauteur relative du houppier : hc/ht)

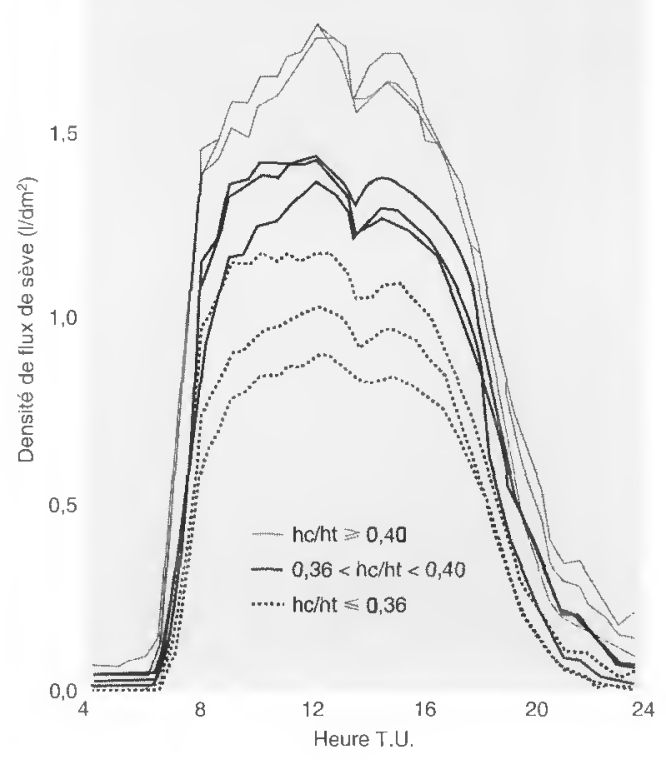

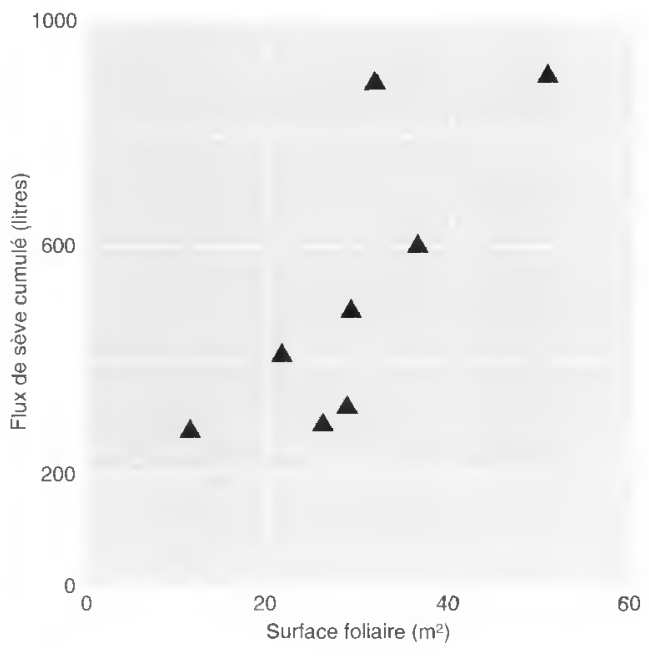

6B Relation entre la densité de flux de sève cumulée sur la saison de végélation (jours 150 à 250) el le statut concurrenliel des arbres mesuré par la hauteur relative du houppier

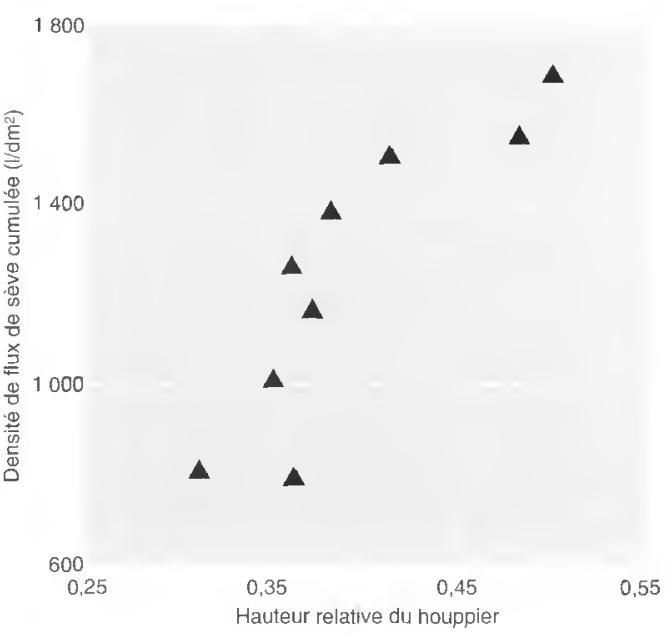


Par ailleurs, aucune régulation stomatique n'a pu être détectée en 1994, malgré les contraintes hydriques assez fortes subies par les arbres : c'est ainsi que la conductance stomatique est restée stable malgré la chute du potentiel hydrique de base des arbres (jusqu'à - 1,5 MPa) au cours de la saison de végétation (figure 7 , ci-dessous). Ce résultat obtenu sur des arbres en conditions naturelles est à rapprocher des résultats expérimentaux obtenus par Aussenac et Lévy en 1983 avec des arbres cultivés en cases de végétation et soumis à la sécheresse, montrant que le Frêne ne régule sa transpiration que très tardivement (Aussenac et Lévy, 1992). Le Frêne apparaît ainsi comme une espèce tolérante à la sécheresse, au regard d'espèces qui régulent leur transpiration très rapidement comme les Pins ou même le Chêne pédonculé (Quercus pedunculata Ehrl.).

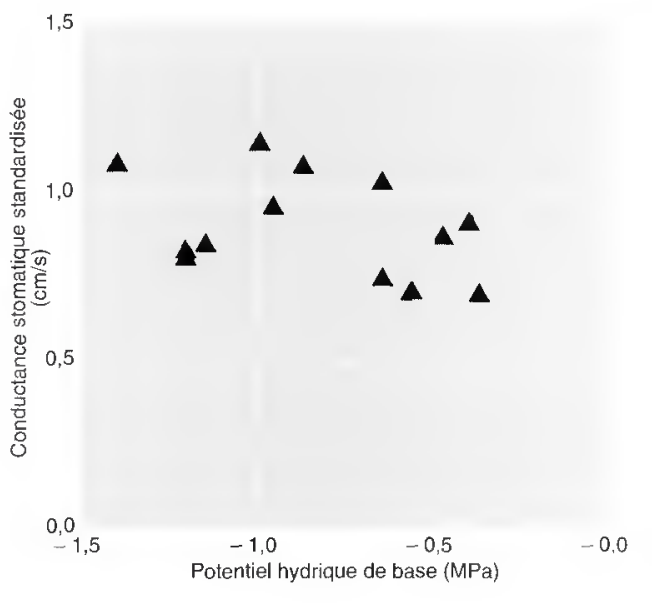

\section{Figure 7}

TRANSPIRATION DU FRÊNE ET STATUT HYDRIQUE : relation enfre la conductance stomalique et le potentiel hydrique de base pour des frênes en condifions naturelles (les mesures de conductance sont standardisées pour une valeur de déficit de saturation de l'air de $1 \mathrm{kPa}$ )

\section{CONCLUSIONS}

L'expérimentation de suivi de la croissance de frênes de divers statuts concurrentiels, mise en place en forêt d'Amance, a permis dans un premier temps d'établir que le volume foliaire utilisé dans le modèle de croissance - estimé à partir des dimensions du houppier et de l'accroissement en hauteur annuel des frênes - était étroitement relié à la biomasse foliaire mesurée (proportionnalité). Par ailleurs, la relation établie entre l'accroissement volumique annuel de la tige, la biomasse foliaire et l'indice foliaire est concordante avec celle obtenue à partir d'analyses rétrospectives d'arbres abattus, d'âges et de statuts concurrentiels variés (Ottorini et al., 1996) ; en effet, dans ces relations, le volume foliaire peut être remplacé par la biomasse foliaire et le rapport du volume foliaire à la surface de la tige (élevée à la puissance 1,5) par l'indice foliaire, compte tenu de la relation linéaire établie entre ces deux grandeurs (Le Goff et Ottorini, 1996).

La croissance volumique des arbres apparaît donc directement liée à la quantité de feuillage qu'ils portent, mais aussi à la densité de feuillage dans le houppier qui semble varier de façon particufière avec le statut concurrentiel des frênes. Les études en cours sur la répartition du feuillage dans le houppier, ses caractéristiques et son fonctionnement devraient permettre de mieux comprendre les variations de "rendement" des arbres (efficience foliaire) observées avec la densité de feuillage (LAI) et avec le statut concurrentiel des arbres. La régulation de la transpiration due aux stress hydriques ne semble pas être en cause ici puisque les arbres ont continué à transpirer normalement. Cependant, des variations de la transpiration du feuillage et de son efficience photosynthétique en fonction de la position dans le houppier ont pu être d'ores et déjà mises en évidence (Peiffer, 1995). Le modèle de croissance "dendrométrique" actuel pourra donc vraisemblablement être enrichi par la prise en compte de la répartition de la biomasse foliaire dans le houppier et de ses caractéristiques de fonctionnement (eificience photosynthétique en particulier). 


\section{Perspectives}

Par ailleurs, les effets des variations climatiques annuelles observées pendant la durée de l'expérimentation (au moins trois ans) sur la croissance des arbres pourront être analysés et pris en compte dans le modèle de croissance (cf. figure 1, p. 157) : effets sur l'élongation de la tige et des branches, sur la quantité de feuillage produite annuellement et sur le fonctionnement hydrique et photosynthétique des arbres.

N. LE GOFF - J.-M. OTTORINI

Unité Croissance, Production et Qualité des Bois INRA

F-54280 CHAMPENOUX
A. GRANIER

Unité Écophysiologie forestière

INRA

F-54280 CHAMPENOUX
Karine LE GALL

Unité Écophysiologie forestière

INRA

F.54280 CHAMPENOUX
Marianne PEIFFER

Unitè Écophysiologie torestière

INRA

F-54280 CHAMPENOUX

\section{Remerciements}

Les mesures dendrométriques de terrain et de laboratoire effectuées pour cette étude en 1993 ont étè conduites par R. Canta avec l'aide de F. Pierson ; l'appui de R. Canta dans la conception et la réalisation des filets pour le recueil des feuilles sur les frênes a aussi été déterminant pour ce travail. L'installation du dispositif ainsi que la mise en place des filets sur les arbres ont de plus bénéficié de l'appui technique de $B$. Clerc et $F$. Willm (Équipe Bioclimatologie et Écophysiologie), D. Himbert (Équipe Sol et Nutrition), L. Garros, P. Garnier et G. Thiriet (Équipe Croissance et Production). Le câblage pour le transfert et le traitement en continu des données microclimatiques et des mesures de flux de sève sont dus à P. Gross (Équipe Bioclimatologie et Écophysiologie). Les mesures écophysiologiques de terrain ont été assurées pour l'essentiel par K. Le Gali et M. Peiffer durant leur stage en 1994 et 1995, avec l'aide technique de B. Clerc et F. Willm. Cette étude a fait l'objet d'une aide incitative de l'INRA (AIP) et bénéficie d'une mise à disposition par l'Office national des Forêts du site expérimental en forêt d'Amance.

\section{BIBLIOGRAPHIE}

AUSSENAC (G.), LÉVY (G.). - Les Exigences en eau du Frêne (Fraxinus excelsior L.). - Revue forestière française, vol. XLIV, ñ spécial "Les feuillus précieux", 1992, pp. 32-38.

CLUZEAU (C.), LE GOFF (N.), OTTORINI (J.-M.). - Development of primary branches and crown profile of Fraxinus excelsior $\mathrm{L}$. - Canadian Journal of Forest Research, vol. 24, $\mathrm{n}^{\circ}$ 12, 1994, pp. 2315-2323.

LE GALL (K.). - Fonctionnement hydrique et croissance du Frêne (Fraxinus excelsior L.) en peuplement. Champenoux: INRA-Nancy, 1994 - 25 p. + annexes (Rapport de stage de maîtrise). 
LE GOFF (N.), OTTORINI (J.-M.). - Crown development and growth of beech (Fagus silvatica L.) after thinning in an experimental stand. In : Genetics and Silviculture of Beech. Proceedings from the $5^{\text {th }}$ Beech Symposium of the IUFRO Project Group P1.10-00, 19-24 September 1994, Mogenstrup, Danemark. - Forskningsserien, $n^{\circ} 11,1995$, pp. 257268.

LE GOFF (N.), OTTORINI (J.-M.). - Leaf development and stem growth of ash (Fraxinus excelsior L.), as affected by tree competitive status. - Journal of Applied Ecology, 1996 (sous presse)

LE GOFF (N.), OTTORINI (J.-M.). - Programme d'étude de la croissance et du développement du Frêne en peuplement par la modélisation et la simulation. - Champenoux : INRA Nancy - Station de Sylviculture et de Production, juin 1990. - 16 p. + annexes (Document interne).

LÉVY (G.), LE GOFF (N.), LEFĖVRE (Y.), GARROS (L.). — Les Exigences stationnelles du Frêne dans le Nord et le NordEst de la France. - Revue forestiére française, vol. XLIV, $n^{\circ}$ spécial "Les feuillus précieux", 1992, pp. 20-26.

OTTORINI (J.-M.). - Growth and development of individual Douglas-fir in stands for applications to simulation in silviculture. - Annales des Sciences forestières, vol. 48, 1991, pp. 651-666.

OTTORINI (J.-M.), LE GOFF (N.), CLUZEAU (C.). - Relationships between crown dimensions and stem development in Fraxinus excelsior L. - Canadian Journal of Forest Research, 1996 (sous presse).

PEIFFER (M.). - Analyse de la croissance, du fonctionnement hydrique, photosynthétique et de l'efficience de l'eau de Frênes de statuts concurrentiels variés. - Nancy: INRA ; Université de Nancy I, 1995 - 20 p. + annexes (DEA de Biologie forestière). 